\title{
Comorbidades entre Pacientes com Artrite Reumatoide e sua Associação com a Descompensação Clínica
}

\author{
Ana Cláudia de Carvalho Fernandes, ${ }^{1}$ Thuane Sales Gonçalves, ${ }^{2}$ \\ Hágabo Mathyell Silva, Carina de Morais Neves, Isabela Viana Oliveira, \\ Djenane Ramalho de Oliveira, Adriana Maria Kakehasi, \\ Maria Raquel Costa Pinto, Mariana Martins Gonzaga do Nascimento
}

\section{RESUMO}

A artrite reumatoide (AR) é uma doença inflamatória crônica que acomete as articulações e evolui com incapacidade funcional. Pacientes com AR frequentemente apresentam comorbidades que podem influenciar a evolução clínica da doença. Objetivo: avaliar a associação entre comorbidades e o perfil de atividade da AR entre pacientes em acompanhamento ambulatorial em um hospital universitário público. Métodos: trata-se de um estudo transversal que coletou dados demográficos sobre doenças, hábitos de vida e perfil da AR de uma amostra representativa de pacientes diagnosticados com artrite reumatoide deste ambulatório. Realizou-se uma análise descritiva e análises uni e multivariadas para avaliar fatores associados com a variável dependente DAS28 (Disease Activity Score 28). Resultados: foram incluídos 201 pacientes. Destes, $172(85,6 \%)$ eram mulheres e a média de idade foi de 60,3 \pm 11,5. Sete pacientes (3.5\%) eram obesos, $17(8.5 \%)$ tabagistas, $106(52,7 \%)$ hipertensos, $38(18,9 \%)$ diabéticos e $72(35,8 \%)$ apresentavam dislipidemias. Foi identificado na amostra um índice de comorbidade funcional médio de 2,3 $\pm 1,19$, e uma média de 4,2 $\pm 2,2$ doenças por paciente. Idade maior ou igual a 60 anos (OR=0,40; IC95\% 0,21-0,74) e ser diabético (OR=2,96; IC95\% 1,30-6,74) mostraram-se associadas com o DAS28 moderado/alto. Conclusão: a presença de comorbidades apresentou-se frequente na população estudada, e idade igual ou superior a 60 anos mostrou-se associada negativamente, enquanto o diagnóstico de diabetes mellitus (DM) apareceu associado positivamente à atividade da doença moderada à alta. Os achados reforçam a necessidade de monitorização da DM entre esses pacientes, especialmente os mais jovens.

Palavras-chave: Artrite reumatoide; comorbidade; gerenciamento clínico; Diabetes Mellitus; idade.

\section{COMORBIDITIES AMONG PATIENTS WITH RHEUMATOID ARTHRITIS} AND THEIR ASSOCIATION WITH CLINICAL DECOMPENSATION

\section{ABSTRACT}

Rheumatoid arthritis (RA) is a inflammatory, chronic disease, which can bring several consequences to the individual. Patients with RA can present several comorbidities that may influence the clinical evolution of RA. Objective: evaluate the association between comorbidities and the RA activity profile among patients attending a rheumatology clinic of a public university hospital. Methods: this is a cross-sectional study, which collected demographic, diseases, lifestyle habits and RA profile data from a representative sample of patients with RA attending the outpatient clinic. A descriptive analysis of these data was performed, as well as univariate and multivariate analyzes to evaluate factors associated with the DAS28 (Disease Activity Score 28). Results: two hundred and one patients were included, a female majority $(85.6 \% ; n=172)$ was detected and the mean age was $60.3 \pm 11.5$. Seven patients $(3.5 \%)$ were obese, 17 (8.5\%) smokers, 106 (52.7\%) hypertensive, 38 (18.9\%) diabetic and 72 (35.8\%) had dyslipidemia. A mean functional comorbidity index of $2.3 \pm 1.19$ and a mean of $4.2 \pm 2,2$ diseases per patient were identified. Age equal or greater than 60 years (OR $=0.40,95 \% \mathrm{Cl} 0.21-0.74)$ and be diabetic $(\mathrm{OR}=2.96,95 \% \mathrm{Cl}, 30-6,74)$ were statistically significantly associated with moderate or high DAS28. Conclusion: the presence of comorbidities was frequent among patients with RA. Furthermore, age equal to or greater than 60 years was negatively associated, and a diagnosis of diabetes mellitus (DM) positively associated with moderate to high disease activity according. The findings reinforce the need for DM monitoring among these patients, especially the younger ones. Keywords: Arthritis rheumatoid; comorbidity; disease management; Diabetes mellitus; age.

RECEBIDO EM: 25/5/2021

MODIFICAÇÕES SOLICITADAS EM: 5/7/2021

ACEITO EM: 7/7/2021

\footnotetext{
1 Universidade Federal de Minas Gerais. Belo Horizonte/MG, Brasil.

2 Autora correspondente. Escola Paulista de Medicina - Unifesp. Rua Botucatu, n 740 - Vila Clementino. São Paulo/SP, Brasil. CEP 04023-062. http://lattes.cnpq.br/9997281751331135. https://orcid.org/0000-0003-1163-2225. thuanesg@gmail.com
} 


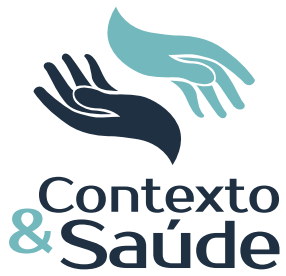

\section{INTRODUÇÃO}

A artrite reumatoide (AR) é uma doença inflamatória crônica, autoimune, sistêmica e progressiva, caracterizada por destruição tecidual, tanto da cartilagem articular quanto do osso subjacente. Cursa com dor, edema e deformações articulares, comprometendo a mobilidade física e a capacidade funcional do paciente $^{1,2}$. A doença pode ocorrer em qualquer idade e em ambos os sexos, embora seja mais comum em mulheres na faixa etária entre 30 e 50 anos $^{3}$.

A prevalência mundial da AR é de aproximadamente $1 \%{ }^{3}$ e, no Brasil, estima-se que a prevalência seja de $0,2-1 \%^{4}$. Um ponto desafiador no que se refere à AR é o fato de os seus pacientes geralmente apresentarem várias outras doenças, que podem decorrer da própria fisiopatologia reumática da condição, ou até mesmo de seu tratamento, dificultando o alcance da remissão pelo paciente. Além disso, doenças como hipertensão arterial sistêmica, dislipidemia e diabetes mellitus são frequentes, aumentando o risco cardiovascular desses pacientes ${ }^{5}$, que já tende a ser elevado devido ao processo inflamatório crônico da $A R^{6}$.

As comorbidades, entretanto, muitas vezes não são devidamente avaliadas, e algumas diretrizes desconsideram que a presença de comorbidades é, atualmente, mais uma regra do que uma exceção entre pacientes com AR. Neste contexto, a European Alliance of Associations for Rheumatology (Eular)7, a Sociedade Brasileira de Reumatologia ${ }^{5}$ e Loza et al. (2014) ${ }^{8}$ propuseram recomendações para a detecção, manejo e prevenção de comorbidades específicas, incluindo a avaliação quanto ao risco cardiovascular pelo menos uma vez a cada cinco anos ${ }^{9}$. As múltiplas doenças apresentadas pelo paciente com AR também devem ser levadas em consideração para definição e ajuste do tratamento, posto que influenciam na qualidade de vida e no prognóstico do paciente e na efetividade do tratamento da AR.

Por conseguinte, o objetivo do presente estudo foi avaliar a associação entre doenças e outros problemas de saúde e o perfil dos índices de atividade da AR em pacientes atendidos em um ambulatório de reumatologia de um hospital público universitário.

\section{MATERIAIS E MÉTODOS}

Trata-se de um estudo transversal do perfil de doenças crônicas apresentadas por pacientes com AR e sua associação com o índice de atividade de doença. O presente estudo foi desenvolvido no Ambulatório de Artrite Reumatoide do Hospital das Clínicas da Universidade Federal de Minas Gerais.

Amostra e coleta de dados: em novembro de 2017, quando o presente estudo começou a ser desenvolvido, o serviço de reumatologia do ambulatório atendia 395 pacientes diagnosticados com AR. Dessa forma, a partir dessa população, uma amostra mínima de 196 pacientes foi definida, considerando um nível de significância de 95,0\% e erro amostral de 5\%.

Foram coletados dados de 201 pacientes no período de 1으 de novembro de 2017 a 11 de maio de 2018, configurando uma amostra de conveniência estatisticamente representativa da população. Todos os dados foram coletados dos prontuários dos pacientes e organizados em um banco de dados no software Microsoft Excel ${ }^{\circledR}$, versão 2016.

Editora Unijuí - Revista Contexto \& Saúde - ISSN 2176-7114 - v. 21, n. 44, out./dez. 2021 


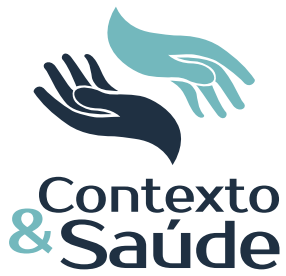

Variáveis coletadas: foram coletados dados demográficos (idade e sexo) e dados sobre a AR e outras doenças e problemas de saúde. Coletou-se o índice combinado de atividade de doença DAS28 (Disease Activity Score 28), calculado utilizando a Proteína C Reativa (PCR) disponível para 181 pacientes (erro amostral de 5,4\%, considerando um nível de significância de 5,0\% para prevalências entre 1,0 e 50,0\%). Para classificação desse índice, foram utilizados os pontos de corte para remissão, baixa, moderada e alta atividade, definidos pela Liga Europeia Contra Artrite Reumatoide ${ }^{10}$.

Também foram identificadas as outras doenças e problemas de saúde documentados no prontuário do paciente. Estes foram classificados como "hipercomorbidades" (doença associada à AR frequentemente) e hipocomorbidades (doença associada à AR em baixa frequência), conforme proposto por Dougados (2016) ${ }^{11}$, ou "outras doenças", quando não se encaixavam nos outros estratos. Também foi calculado o Índice de Comorbidade Funcional (ICF) desenvolvido por Groll et al. $(2005)^{12}$. Trata-se de um escore simples, cujo cálculo leva em consideração a presença de doenças e outros fatores de risco em saúde; podendo variar de zero a 18, sendo que zero indica ausência de qualquer doença ou fatores do índice, e 18 indica presença de todas as doenças ou fatores do índice, que são 18 no total.

Análise estatística: foi realizada uma análise descritiva dos dados com determinação das frequências absolutas e relativas das variáveis qualitativas e média, desvio-padrão, mediana, mínimo e máximo das variáveis quantitativas. Também foram realizadas análises univariadas e multivariadas para avaliar fatores associados com a variável dependente DAS28 moderado ou alto, ou seja, valores de DAS28 superiores a 3,2.

Foram consideradas as seguintes variáveis: sexo (feminino versus masculino); idade (18 a 60 anos versus $\geq 60$ anos); ICF (zero a 2 versus $\geq 3$; dicotomização de acordo com a mediana da variável); anos com artrite reumatoide (zero a 15 versus $\geq 16$; dicotomização de acordo com a mediana da variável); tabagismo (sim versus não); e obesidade (sim versus não).

Adicionalmente também foram incluídas como variáveis independentes diferentes doenças identificadas na amostra e consideradas, pela literatura, comorbidades relacionadas à $A R$ ou ao tratamento da $A R^{8,11}$ : diabetes, hipertensão, dislipidemia, doença cardiovascular aterosclerótica (incluídos pacientes com histórico de angina, infarto agudo do miocárdio, acidente vascular cerebral isquêmico ou doença aterosclerótica subclínica), insuficiência cardíaca, osteoporose, doença do trato gastrointestinal (incluídos pacientes com histórico de qualquer doença do trato gastrointestinal superior ou inferior), doenças pulmonares (incluídos pacientes com qualquer doença pulmonar, sendo a doença pulmonar obstrutiva crônica e asma as mais comuns), infecções, distúrbio de humor ou ansiedade (incluídos pacientes com diagnóstico de qualquer distúrbio de humor e/ou ansiedade), câncer (incluídos pacientes com qualquer tipo de câncer).

Para as análises univariadas utilizou-se o teste de qui-quadrado de Pearson ou o teste exato de Fisher, quando o valor esperado de uma ou mais células for cinco ou menos. Variáveis independentes com $p<0,20$ nas análises univariadas foram incluídas no modelo multivariado que foi calculado por meio de regressão logística stepwise. Foi utilizado o teste Hosmer-Lemeshow para avaliar a

Editora Unijuí - Revista Contexto \& Saúde - ISSN 2176-7114 - v. 21, n. 44, out./dez. 2021 
qualidade de ajuste do modelo multivariado. As análises univariada e multivariada foram baseadas no odds ratio (OR) e no respectivo intervalo de confiança de $95 \%$, estimados por regressão logística. O nível de significância de $5 \%$ foi o critério adotado para identificar as características independentemente associadas à variável dependente. Para todas as análises foi empregado o pacote estatístico Stata ${ }^{\circledR}$, versão 13 (Stata Corp., College Station, Estados Unidos).

Aspectos éticos: o presente projeto está inserido na pesquisa intitulada "Resultados clínicos, econômicos, aspectos humanísticos, culturais e educacionais de serviços de gerenciamento da terapia medicamentosa no Sistema Único de Saúde", aprovada pelo Comitê de Ética em Pesquisa da UFMG (COEP) no dia 28 de maio de 2014, sob registro CAAE-25780314.4.0000.5149. Como foram coletados dados secundários não foi necessária a aplicação do Termo de Consentimento Livre Esclarecido (TCLE).

\section{RESULTADOS}

Nesta pesquisa, a média de idade foi de 60,3 $\pm 11,5$ anos (mediana $=$ 60; mínimo $=27 ;$ máximo $=88$ anos) e detectou-se que 172 pacientes eram do sexo feminino $(85,6 \%)$. Sete pacientes eram obesos $(3,5 \%)$ e 17 eram tabagistas $(8,5 \%)$. Foi identificado na amostra um ICF médio de 2,3 $\pm 1,19$ (mediana $=2$; mínimo = $1 ;$ máximo = 6).

Além da AR, 97,5\% dos pacientes ( $n=196)$ apresentavam pelo menos uma outra doença ou problema de saúde. Foi detectada uma média de 4,2 $\pm 2,2$ doenças por paciente $($ mediana $=4 ;$ mínimo $=0 ;$ máximo $=10)$. A doença mais prevalente foi a hipertensão arterial sistêmica (HAS) (52,7\%; n=106). Na Tabela 1 são apresentadas as frequências das comorbidades relacionadas à AR identificadas na amostra.

Tabela 1 - Frequência das comorbidades identificadas na amostra

\begin{tabular}{lll}
\hline \multicolumn{1}{c}{ Comorbidades } & $\mathbf{n}$ & \% \\
\hline Hipercomorbidades & 58 & 28,9 \\
\hline Osteoporose & 45 & 22,4 \\
Infecções & 36 & 17,9 \\
Doença do trato gastrointestinal & 34 & 16,9 \\
Distúrbios de humor/ansiedade & 21 & 10,5 \\
Doenças cardiovasculares ateroscleróticas & & \\
\hline Hipocomorbidades & 3 & 1,5 \\
\hline Câncer de cólon & 2 & 1,0 \\
Doença de Alzheimer & & \\
\hline Outras comorbidades & 106 & 52,7 \\
\hline Hipertensão & 72 & 35,8 \\
Dislipidemia & 38 & 18,9 \\
Diabetes & 34 & 16,9 \\
Doença pulmonar & 12 & 6,0 \\
Outros cânceres & 12 & 6,0 \\
Insuficiência cardíaca &
\end{tabular}

Editora Unijuí - Revista Contexto \& Saúde - ISSN 2176-7114 - v. 21, n. 44, out./dez. 2021 
Tanto na análise univariada quanto na análise multivariada, apenas as variáveis idade e diabetes mellitus mostraram-se associadas de forma estatisticamente significativa com o DAS28 moderado ou alto (Tabela 2).

Tabela 2 - Resultados das análises univariada e multivariada das características associadas ao DAS28 (índice combinado de atividade de doença do tipo Disease Activity Score 28) moderado ou alto

\begin{tabular}{|c|c|c|c|c|c|c|}
\hline \multirow[b]{2}{*}{ Variáveis } & \multicolumn{2}{|c|}{ DAS28* } & \multicolumn{2}{|c|}{ Análise Univariada } & \multicolumn{2}{|c|}{ Análise Multivariada } \\
\hline & $\begin{array}{c}\text { Remissão ou } \\
\text { baixa } \\
\text { n (\%) }\end{array}$ & $\begin{array}{c}\text { Moderada ou } \\
\text { alta } \\
\text { n (\%) }\end{array}$ & OR (IC95\%)** & $\mathrm{p}$-valor*** & OR (IC95\%)" & p-valor \\
\hline \multicolumn{7}{|l|}{ Idade (anos) } \\
\hline 18 a 59 & $39(44,8)$ & $48(55,2)$ & 1,0 & - & 1 & - \\
\hline 60 ou mais & $60(64,5)$ & $33(35,5)$ & $0,45(0,24-0,81)$ & 0,008 & $0,40(0,21-0,74)$ & 0,004 \\
\hline \multicolumn{7}{|l|}{ Sexo } \\
\hline Feminino & $88(55,0)$ & $72(45,0)$ & 1,0 & - & - & - \\
\hline Masculino & $13(59,1)$ & $9(40,9)$ & $0,75(0,30-1,91)$ & 0,717 & - & - \\
\hline \multicolumn{7}{|c|}{ Anos com artrite reumatoide } \\
\hline Zero a 15 & $44(57,1)$ & $33(42,9)$ & 1,0 & - & - & - \\
\hline 16 ou mais & $56(55,5)$ & $45(44,5)$ & $1,10(0,61-2,01)$ & 0,821 & - & - \\
\hline \multicolumn{7}{|l|}{ Tabagismo } \\
\hline Não & $93(56,0)$ & $73(44,0)$ & 1,0 & - & - & - \\
\hline Sim & $8(50,0)$ & $8(50,0)$ & $1,29(0,46-3,61)$ & 0,643 & - & - \\
\hline \multicolumn{7}{|l|}{ Obesidade } \\
\hline Não & $99(56,6)$ & $76(43,4)$ & 1,00 & - & - & - \\
\hline Sim & $2(28,6)$ & $5(71,4)$ & $3,30(0,62-17,48)$ & 0,144 & - & - \\
\hline \multicolumn{7}{|l|}{ ICF"\# } \\
\hline Zero a 2 & $64(57,7)$ & $47(42,3)$ & 1,00 & - & - & - \\
\hline 3 ou mais & $37(52,1)$ & $34(47,9)$ & $1,10(0,85-1,41)$ & 0,463 & - & - \\
\hline \multicolumn{7}{|l|}{ Hipertensão } \\
\hline Não & $48(55,8)$ & $38(44,2)$ & 1,00 & - & - & - \\
\hline Sim & $53(55,2)$ & $43(44,8)$ & $1,05(0,58-1,89)$ & 0,935 & - & - \\
\hline \multicolumn{7}{|c|}{ Diabetes mellitus } \\
\hline Não & $89(59,7)$ & $60(40,3)$ & 1,00 & - & 1,00 & - \\
\hline Sim & $12(36,6)$ & $21(63,6)$ & $2,47(1,12-5,43)$ & 0,015 & $2,96(1,30-6,74)$ & 0,010 \\
\hline \multicolumn{7}{|l|}{ Dislipidemia } \\
\hline Não & $62(53,0)$ & $55(47,0)$ & 1,00 & - & - & - \\
\hline Sim & $39(60,0)$ & $26(40,0)$ & $0,76(0,41-1,42)$ & 0,362 & - & - \\
\hline \multicolumn{7}{|c|}{ Distúrbio do humor ou ansiedade } \\
\hline Não & $85(57,4)$ & $63(42,6)$ & 1,00 & - & - & - \\
\hline Sim & $16(48,5)$ & $17(51,5)$ & $1,43(0,67-3,05)$ & 0,349 & - & - \\
\hline \multicolumn{7}{|c|}{ Doença cardiovascular aterosclerótica } \\
\hline Não & $94(57,0)$ & $71(43,0)$ & 1,00 & - & - & - \\
\hline Sim & $7(43,8)$ & $9(56,2)$ & $1,70(0,60-4,79)$ & 0,387 & - & - \\
\hline \multicolumn{7}{|c|}{ Insuficiência cardíaca } \\
\hline Não & $96(55,8)$ & $76(44,2)$ & 1,00 & - & - & - \\
\hline Sim & $5(50,0)$ & $5(50,0)$ & $1,28(0,36-4,58)$ & 0,719 & - & - \\
\hline \multicolumn{7}{|l|}{ Osteoporose } \\
\hline Não & $67(51,5)$ & $63(48,5)$ & 1,00 & - & - & - \\
\hline Sim & $34(65,4)$ & $18(34,6)$ & $0,57(0,29-1,12)$ & 0,090 & - & - \\
\hline \multicolumn{7}{|c|}{ Doença do trato gastrointestinal } \\
\hline Não & $81(53,3)$ & $71(46,7)$ & 1,00 & - & - & - \\
\hline Sim & $20(66,7)$ & $10(33,3)$ & $0,58(0,25-1,32)$ & 0,178 & - & - \\
\hline \multicolumn{7}{|c|}{ Doença pulmonar } \\
\hline Não & $88(56,8)$ & $67(43,2)$ & 1,00 & - & - & - \\
\hline Sim & $13(48,2)$ & $14(51,8)$ & $1,44(0,63-3,26)$ & 0,405 & - & - \\
\hline Câncer & & & & & & \\
\hline Não & $95(56,2)$ & $74(43,8)$ & 1,00 & - & - & - \\
\hline $\operatorname{sim}$ & $6(46,1)$ & $7(53,9)$ & $1,51(0,49-4,71)$ & 0,482 & - & - \\
\hline Infecções gra & & & & & & \\
\hline Não & $78(56,1)$ & $61(43,9)$ & 1,00 & - & - & - \\
\hline Sim & $23(53,5)$ & $20(46,5)$ & $1,06(0,53-2,11)$ & 0,762 & - & - \\
\hline
\end{tabular}

* DAS18 = Disease Activity Score 28; moderada a alta se superior a 3.2

** Odds ratio (Intervalo de Confiança de 95\%) - estimados por regressão logística

*** Estimado pelo teste de qui-quadrado de Pearson

\# Estimado por regressão logística stepwise; estatisticamente significativo quando < 0,05

\#\#ICF = Índice de Comorbidade Funcional (ICF); desenvolvido por Groll et al. $(2005)^{12}$. 


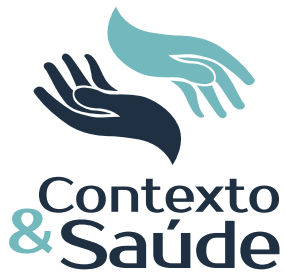

\section{DISCUSSÃO}

A alta prevalência de mulheres na população estudada $(85,6 \%)$, bem como a média de idade encontrada (60,3 anos), corrobora os dados descritos na literatura ${ }^{13}$. Neste estudo, a variável idade mostrou-se negativamente associada e de forma independente à atividade da doença moderada ou alta, calculada pelo DAS28 (OR 0,40; IC95\% 0,21-0,74). Cento e noventa e seis pacientes $(97,5 \%)$ apresentavam pelo menos uma outra doença além da AR, com uma média de 4,2 $\pm 2,2$ doenças por paciente, confirmando a prevalência relativamente alta de comorbidades entre pacientes com AR. Dentre essas, a DM apresentou prevalência de $18,9 \%$ na população estudada e mostrou-se independentemente associada à detecção de atividade da doença moderada ou alta com força de associação considerável (OR = 2,96; IC95\% 1,30-6,74).

Dos pacientes com idade igual ou superior a 60 anos, $64,5 \%$ apresentavam remissão ou baixa atividade da doença, contra 35,5\% que apresentavam atividade moderada ou alta. Já entre os pacientes com idade inferior a 60 anos, $44,8 \%$ apresentavam remissão ou baixa atividade da doença, contra $55,2 \%$ que apresentavam atividade alta ou moderada da doença, mostrando uma relação inversa entre idade e atividade da AR. Esses resultados diferem daqueles expostos em outros estudos, em que a idade mais avançada representa fator de risco independente para não remissão da $A R^{14}$. A associação negativa encontrada pode estar relacionada ao cuidado mais intenso direcionado às populações mais envelhecidas, levando a um quadro de menor atividade da doença entre os pacientes idosos. Nosso desenho de estudo, entretanto, não permite fazer maiores inferências, sendo necessários mais estudos para elucidar essa associação.

Sobre as comorbidades, apesar de esperada, a prevalência encontrada neste estudo foi superior à identificada na África do Sul $(69,3 \%)^{15}$ e no norte da Suécia $(53,2 \%)^{16}$. Essa alta prevalência também pode ser explicada considerando a média de idade da população. De modo geral, indivíduos idosos (60 anos ou mais) apresentam diversas doenças crônicas, com destaque para $\mathrm{HAS}^{15,17}$, presente em $52,7 \%$ da população estudada, e igualmente superior à prevalência identificada por outros estudos entre pacientes portadores de AR, que variou de 20,7 a $47,5 \%{ }^{15,16,18-22}$.

Atualmente, está bem estabelecido que pacientes com AR apresentam maior morbimortalidade cardiovascular em comparação com a população geral. Em adição, a prevalência e gravidade de diversos fatores de risco cardiovasculares tradicionais, entre eles HAS e dislipidemia, são maiores nesses pacientes ${ }^{23}$. Na população estudada, além de uma alta prevalência para HAS, observou-se também alta prevalência para dislipidemias $(35,8 \%)$, estando de acordo com o que é descrito na literatura ${ }^{24}$.

Outro importante fator de risco para doenças cardiovasculares e mortalidade cardiovascular entre pessoas com AR é o diabetes mellitus ${ }^{25,26}$ que, em nosso estudo, apresentou prevalência superior ao estimado para população mundial $(8,8 \%)$ e brasileira $(8,7 \%)$, bem como ao detectado em outros estudos de pacientes com $A R$, que variou de $4 \%$ a $15 \%$ 15,16,18-22.

Editora Unijuí - Revista Contexto \& Saúde - ISSN 2176-7114 - v. 21, n. 44, out./dez. 2021 
Pacientes com AR podem desenvolver resistência à insulina, associada ao processo inflamatório da própria doença, caracterizado pela presença de citocinas como TNF- $\alpha$, IL-1 e IL-6. Essa resistência à insulina pode explicar a significativa prevalência de DM nesses pacientes ${ }^{27-29}$. Por outro lado, também devido ao caráter inflamatório da $\mathrm{DM}^{30}$, já foi demonstrado o potencial de aumento da incidência de AR entre pacientes com DM estabelecida ${ }^{31-34}$.

Algumas pesquisas já demonstraram uma relação inversa entre escores de atividade da doença na AR e função de células beta ${ }^{35}$. Ao nosso conhecimento, contudo, inexiste outro trabalho na literatura que demonstre associação similar à investigada neste estudo. Marques et al. $(2015)^{36}$, em um estudo com delineamento semelhante, identificaram a associação entre ICF e atividade da doença (DAS28) em modelo multivariado, o que, apesar de avaliado no presente estudo, não foi identificado.

A coleta de dados secundários em prontuário foi uma das limitações deste trabalho. Apesar de existir um padrão específico para documentação tanto de doenças (que podem ser identificadas por diagnóstico médico ou relato do paciente) quanto de parâmetros de atividade da doença (ausentes para alguns pacientes da amostra) neste ambulatório, por meio de prontuário estruturado, tais dados, muitas vezes, não estavam preenchidos adequadamente.

A avaliação da associação em delineamento transversal e com amostra somente de um ambulatório de reumatologia também traz limitações adicionais ao estudo. Os resultados identificados, no entanto, reforçam a forte inter-relação entre o DM e AR já estabelecida na literatura, bem como a necessidade de controle adequado tanto do DM quanto da $A R$, promovendo sucesso terapêutico mútuo nessas comorbidades e na prevenção de eventos cardiovasculares.

Deve-se destacar que o controle adequado das comorbidades associadas à $A R$ nem sempre é realizado, conforme demonstrado em um estudo americano que incluiu mais de 16 mil pacientes com AR e pelo menos uma segunda doença concomitante. Na amostra analisada, $90 \%$ dos pacientes apresentavam doenças cardiovasculares, $64 \%$ hiperlipidemia e $46 \%$ diabetes. Observou-se, entretanto, que foi realizado, em apenas $63 \%$ dos pacientes, o teste lipídico anual, apesar de essa ser uma estratégia recomendada para monitorar e reduzir a morbimortalidade por comorbidades em pacientes com AR e doença cardiovascular, diabetes ou hiperlipidemia concomitante ${ }^{24}$.

Diante isso, propõe-se uma visão ampla e holística no manejo da AR, devendo ser encorajado que profissionais, mesmo que especializados em reumatologia, atentem para a necessidade de avaliar e controlar comorbidades, com destaque para o DM. O manejo das comorbidades é importante para que se obtenha sucesso terapêutico e controle dos índices de atividade da doença, sobretudo no cenário em estudo, em que $60,8 \%$ dos pacientes ainda não se encontram em remissão, de acordo com o DAS28. Neste contexto, destaca-se a relevância do trabalho das equipes multidisciplinares e de profissionais de diferentes níveis de atenção que possam garantir uma abordagem global como estratégia para o controle da doença ${ }^{8,11,13}$. 


\section{CONCLUSÃO}

Em conclusão, foi possível evidenciar a frequente presença de comorbidades entre os pacientes com AR. Além disso, a idade igual ou superior a 60 anos mostrou-se associada negativamente e o diagnóstico de DM associado positivamente ao índice de atividade da doença moderado a alto, conforme o DAS28. Tal fato reforça a necessidade de monitorização de DM entre pacientes com AR, com destaque para pacientes mais jovens.

\section{REFERÊNCIAS}

${ }^{1}$ Ministério da Saúde. Portaria Conjunta n 15, de 11 de dezembro de 2017. Aprova o Protocolo Clínico e Diretrizes Terapêuticas da Artrite Reumatoide. Diário Oficial da União. 2017;26 dez.

${ }^{2}$ Goeldner I, Skare TL, Reason ITM, Utiyama SRR. Artrite reumatoide: uma visão atual. Jornal Brasileiro de Patologia e Medicina Laboratorial. 2011;47(5):495-503.

${ }^{3}$ Wasserman AM. Diagnosis and management of rheumatoid arthritis. American Family Physician. 2011;84(11):1245-1252.

${ }^{4}$ Senna ER, De Barros AL, Silva EO, Costa IF, Pereira LV, Ciconelli RM, et al. Prevalence of rheumatic diseases in Brazil: a study using the COPCORD approach. J Rheumatol. 2004 Mar;31(3):594-7.

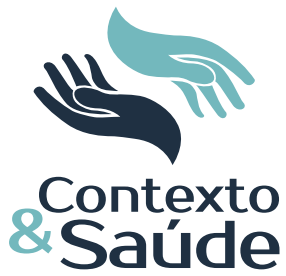

5 Pereira IA. Rheumatoid arthritis: Why to treat only arthritis, knowing that comorbidities are common and determine morbidity and mortality? Rev Bras Reumatol. 2012;52(4):472-3.

${ }^{6}$ Smolen JS, Aletaha D, Barton A, Burmester GR, Emery P, Firestein GS et al. Rheumatoid arthritis. Nat Rev Dis Primers. 2018;4:18001.

${ }^{7}$ Baillet A, Gossec L, Carmona L, Witt Md, van Eijk-Hustings Y, Bertheussen $\mathrm{H}$ et al. Points to consider for reporting, screening for and preventing selected comorbidities in chronic inflammatory rheumatic diseases in daily practice: a EULAR initiative. Ann Rheum Dis. 2016 jun.; 75(6):965-73.

${ }^{8}$ Loza E, Lajas C, Andreu JL, Balsa A, González-Álvaro I, Illera O et al. Consensus statement on a framework for the management of comorbidity and extra-articular manifestations in rheumatoid arthritis. Rheumatology International. 2014;35(3):445-58.

${ }^{9}$ Agca R, Heslinga SC, Rollefstad S, Heslinga M, Mclnnes IB, Peters MJ et al. EULAR recommendations for cardiovascular disease risk management in patients with rheumatoid arthritis and other forms of inflammatory joint disorders: 2015/2016 update. Ann Rheum Dis. 2017 jan.;76(1):17-28.

${ }^{10}$ Aletaha D, Neogi T, Silman AJ, Funovits J, Felson DT, Bingham CO et al. 2010 Rheumatoid arthritis classification criteria: an American College of Rheumatology/European League Against Rheumatism collaborative initiative. Arthritis Rheum. 2010 set.;62(9):2569-81.

${ }^{11}$ Dougados M. Comorbidities in rheumatoid arthritis. Current Opinion In Rheumatology 2016; 28(3):282-8.

12 Groll D, To T, Bombardier C, Wright JG. The development of a comorbidity index with physical function as the outcome. J Clin Epidemiol. 2005 Jun;58(6):595-602.

${ }^{13}$ Sokka T, Toloza S, Cutolo M, Hannu K, Makinen H, Gogus F et al. Women, men, and rheumatoid arthritis: analyses of disease activity, disease characteristics, and treatments in the QUEST-RA study. Arthritis Research \& Therapy. 2009;11(1):R7.

${ }^{14}$ Ito H, Ogura T, Hirata A, Takenaka S, Mizushina K, Fujisawa Y et al. Global assessments of disease activity are age-dependent determinant factors of clinical remission in rheumatoid arthritis. Seminars in Arthritis and Rheumatism. 2017;47(3):310-4.

${ }^{15}$ Olivier N, Burger J, Joubert R, Lubbe M, Cockeran M. Chronic disease list conditions in patients with rheumatoid arthritis in the private healthcare sector of South Africa. Rheumatology International. 2018;38(5):837-44. 
${ }^{16}$ Innala L, Sjoberg C, Moller B, Ljung L, Smedby T, Sodergren A et al. Co-morbidity in patients with early rheumatoid arthritis - inflammation matters. Arthritis Research \& Therapy. 2016;18(1):18-33.

${ }^{17}$ Pimenta FB, Pinho L, Silveira MF, Botelho ACC. Fatores associados a doenças crônicas em idosos atendidos pela Estratégia de Saúde da Família. Ciência \& Saúde Coletiva. 2015; 20(8):2489-98.

${ }^{18}$ Jeong H, Baek SY, Kim SW, Eun YH, Kim IY, Kim H et al. Comorbidities of rheumatoid arthritis: Results from the Korean National Health and Nutrition Examination Survey. Plos One. 2017;12(4):e0176260.

${ }^{19}$ Chandrashekara S, Shobha V, Dharmanand BG, Jois R, Kumar S, Mahendranath KM et al. Comorbidities and related factors in rheumatoid arthritis patients of south India- Karnataka Rheumatoid Arthritis Comorbidity (KRAC) study. Reumatismo. 2017;69(2):4758.

${ }^{20}$ Galarza-Delgado DA, Azpiri-Lopez JR, Colunga-Pedraza IJ, Cárdenas-de-la Garza JÁ, Vera-Pineda $\mathrm{R}$, Wah-Suaréz $\mathrm{M}$ et al. Prevalence of comorbidities in Mexican mestizo patients with rheumatoid arthritis. Rheumatology International. 2017;37(9):1507-11.

${ }^{21}$ Dougados M, Soubrier M, Antunez A, Balint P, Balsa A, Buch MH et al. Prevalence of comorbidities in rheumatoid arthritis and evaluation of their monitoring: results of an international, cross-sectional study (COMORA). Annals Of The Rheumatic Diseases. 2014;73(1):62-8.

${ }^{22}$ Mohammad A, Hartery K, Bond U, Phelan M. Increased occurrence of cardiovascular events and comorbidities in a general rheumatology cohort. Irish Journal Of Medical Science 2010;179(2):273-6.

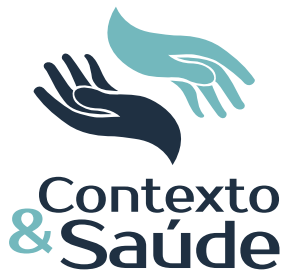

${ }^{23}$ Puttevils D, De Vusser P, Geusens P, Dens J. Increased cardiovascular risk in patients with rheumatoid arthritis: an overview. Acta Cardiologica. 2014;69(2):111-8.

${ }^{24}$ Bartels CM, Kind AJ, Thorpe CT, Everett CM, Cook RJ, McBride PE et al. Lipid Testing in Patients with Rheumatoid Arthritis and Key Cardiovascular-Related Comorbidities: A Medicare Analysis. Seminars In Arthritis And Rheumatism. 2012;42(1):9-16.

${ }^{25}$ Hoek JVD, Roorda LD, Boshuizen HC, Tijhuis GJ, Dekker J, van den Bos GA et al. Trend in and Predictors for Cardiovascular Mortality in Patients with Rheumatoid Arthritis Over a Period of 15 Years: A Prospective Cohort Study. Annals of The Rheumatic Diseases. 2016;74(2):692-3.

${ }^{26}$ Baghdadi LR, Woodman RJ, Shanahan EM, Mangoni AA. The Impact of Traditional Cardiovascular Risk Factors on Cardiovascular Outcomes in Patients with Rheumatoid Arthritis: A Systematic Review and Meta-Analysis. Plos One. 2015;10(2):e0117952.

${ }^{27}$ Nicolau J, Lequerré T, Bacquet H, Vittecoq O. Rheumatoid arthritis, insulin resistance, and diabetes. Joint Bone Spine. 2017;84(4):411-6.

${ }^{28}$ Burska AN, Sakthiswary R, Sattar N. Effects of Tumour Necrosis Factor Antagonists on Insulin Sensitivity/Resistance in Rheumatoid Arthritis: A Systematic Review and Meta-Analysis. Plos One. 2015;10(6).

${ }^{29}$ Dubreuil M, Rho YH, Man A, Zhu Y, Zhang Y, Love TJ et al. Diabetes incidence in psoriatic arthritis, psoriasis and rheumatoid arthritis: a UK population-based cohort study. Rheumatology, Oxford. 2014;53(2):346-52.

${ }^{30}$ Donath MY, Shoelson SE. Type 2 diabetes as an inflammatory disease. Nature Reviews Immunology. 2011;11(2):98-107.

${ }^{31}$ Albrecht K, Luque Ramos A, Hoffmann F, Redeker I, Zink A. High prevalence of diabetes in patients with rheumatoid arthritis: results from a questionnaire survey linked to claims data. Rheumatology, Oxford. 2018 fev. 1;57(2):329-36.

32 Turk SA, van Beers-Tas MH, van Schaardenburg D. Prediction of future rheumatoid arthritis. Rheum Dis Clin North Am [Internet]. 2014;40(4):753-70.

${ }^{33}$ Lu MC, Yan ST, Yin WY, Koo M, Lai NS. Risk of rheumatoid arthritis in patients with type 2 diabetes: A nationwide population-based case-control study. PLoS One. 2014;9(7):510.

${ }^{34}$ Su CC, Chen IC, Young FN, Lian I Bin. Risk of diabetes in patients with rheumatoid arthritis: A 12-year retrospective cohort study. J Rheumatol. 2013;40(9):1513-8. 
${ }^{35}$ Dessein $\mathrm{PH}$, Joffe $\mathrm{BI}$. Insulin resistance and impaired beta cell function in rheumatoid arthritis. Arthritis \& Rheumatism. 2006;54(9):2765-75.

${ }^{36}$ Marques WV, Cruz VA, Rego J, Silva NA. Influência das comorbidades na capacidade funcional de pacientes com artrite reumatoide. Revista Brasileira de Reumatologia. 2015;56(1):14-21.

37 Van Onna M, Boonen A. The challenging interplay between rheumatoid arthritis, ageing and comorbidities. BMC Musculoskelet Disord [Internet]. 2016;17(1):1-9. 